\title{
ATRIBUTOS INDICADORES DA QUALIDADE DO SOLO EM POVOAMENTOS DE EUCALIPTO FERTIRRIGADO NO VALE DO RIO DOCE - MG ${ }^{1}$
}

\section{INDICATORS ATTRIBUTES OF THE QUALITY OF THE SOIL IN SETTLEMENTS OF EUCALYPT FERTIRRIGATED IN THE VALE DO RIO DOCE - MG}

\author{
Crisliane Aparecida Pereira dos SANTOS ${ }^{2}$ \\ Marcos Rogério TÓTOLA ${ }^{3}$ \\ Arnaldo Chaer BORGES ${ }^{4}$ \\ Catarina Megumi KASUYA ${ }^{5}$
}

\begin{abstract}
RESUMO
O objetivo deste trabalho foi acompanhar possíveis mudanças nos atributos indicadores da qualidade do solo em povoamentos de eucalipto, mediante utilização de irrigação e fertirrigação de efluente de uma fábrica de celulose, por meio de um modelo matemático que expressa o Índice de Qualidade do Solo (IQS), utilizando-se atributos microbiológicos e químicos. Foram instalados dois experimentos distintos, ambos dispostos em parcelas subdivididas, sendo um na posição de baixada e o outro na posição de encosta, no município de Belo Oriente - MG, com solos classificados como Neossolo Flúvico e Latossolo Amarelo, respectivamente. Foram considerados os seguintes tratamentos: irrigação até a máxima evapotranspiração (Irrigação), fertirrigação até a máxima evapotranspiração com $100 \%$ da adubação recomendada pela demanda nutricional da planta (Irrigação - Adubação) e fertirrigação com efluente de celulose (Irrigação - Efluente). Amostras de solos de áreas de eucalipto e de duas outras consideradas como referência (mata secundária e pastagem) foram coletadas em 2003, durante duas épocas do ano (períodos chuvoso e seco), sob posição de baixada e de encosta, e analisadas quanto aos atributos microbiológicos e químicos para determinação dos IQS. Os atributos químicos mostraram-se mais sensíveis ao efeito do uso e manejo do solo que ao efeito de época. Os IQS apresentaram comportamento similar entre os tratamentos apenas para a posição de encosta (Latossolo Amarelo), mas não para a de baixada (Neossolo Flúvico).
\end{abstract}

Palavras-chave: Efeito sazonal; posição topográfica; efluente de celulose; atividade microbiana.

\begin{abstract}
The objective of this work was to follow possible changes in the indicators attributes of the quality of soils in settlemens of eucalypt by means of use of irrigation and fertirrigation of effluent of a cellulose plant, by means of a mathematical model that express the Index of Quality of Sol (IQS), using microbiological and chemical attributes. Two experiment distinct were installed, both made use in subdivided parcels, being one in the valley position and the other in the slope position, in the city of Belo Oriente - MG, with classified soils as Fluvic Neosol and Yellow Latosol, respectively. The following treatments were considered: irrigation until the maximum evapotranspiration (Irrigation), fertirrigation until the maximum evapotranspiration with $100 \%$ of the fertilization recommended for the demand nutritional of the plant (Irrigation - Fertilization) and fertirrigation with effluent of cellulose (Irrigation - Effluent). Samples of soils of areas of eucalypt and two others considered as reference (secondary forest and pasture) had been collected in 2003, during two periods of the year (rainy and dry), under valley position and slope, and analyzed how much to the microbiological and chemical attributes for determination of the IQS. The chemical attributes showed more sensible to the effect of the use and management of the soil that time effect. The IQS presented similar behavior between the treatments only for the slope position (Yellow Latosol), but not for that valley position (Fluvic Neosol).

Key-words: Sazonal effect; topographical position; effluents from cellulose; microbial activity.
\end{abstract}

\footnotetext{
${ }^{1}$ Parte da Dissertação de Mestrado da primeira autora, apresentada à Universidade Federal de Viçosa - MG.

${ }^{2}$ Engenheira Agrônoma, Mestre em Microbiologia Agrícola. Coordenadora de Planejamento e Gestão Ambiental do Instituto de Biodiversidade e Desenvolvimento Sustentável do Oeste da Bahia (BIOESTE). Autora para correspondência. Rua D. João VI, n. 447, ap. 401, Edifício Bitencourt, Vila Regina, Barreiras-BA, Brasil. CEP: 47805-330. E-mail: crislianeaparecida@hotmail.com.

${ }^{3}$ Professor Adjunto do Departamento de Microbiologia da Universidade Federal de Viçosa (UFV). CEP: 36571-000, Viçosa - MG, Brasil. E-mail: totola@ufv.br

${ }^{4}$ Professor Titular do Departamento de Microbiologia da Universidade Federal de Viçosa. CEP: 36571-000, Viçosa - MG, Brasil. E-mail: chaer@ufv.br

${ }^{5}$ Professora Adjunto do Departamento de Microbiologia da Universidade Federal de Viçosa. CEP: 36571-000, Viçosa-MG, Brasil. E-mail: mkasuya@ufv.br
} 


\section{INTRODUÇÃO}

A reutilização de águas residuárias provenientes do processo da fabricação de celulose em programas de fertirrigação de povoamentos de eucalipto é considerada uma alternativa de uso e disposição final desse material e, ao mesmo tempo, uma maneira de aumentar a produtividade das áreas fertirrigadas, dada à presença de nutrientes como $\mathrm{Ca}^{2+}, \mathrm{Mg}^{2+}, \mathrm{N}$ e $\mathrm{P}$, em sua composição química. Entretanto, desconhecem-se os efeitos adversos de sua aplicação sobre as características químicas do solo, bem como sobre as populações microbianas ali presentes.

Pesquisas relacionadas à qualidade do solo são necessárias para fornecer informações de manejo e definição da melhor capacidade de uso do solo (Lopes, 2001; Oliveira et al., 2001). A manutenção da qualidade do solo pode ser avaliada quando se consideram os valores de indicadores microbiológicos, químicos e físicos (Kelting et al., 1999; Stenberg, 1999). A interpretação dos resultados em trabalhos de avaliação da qualidade de solos pode ser realizada mediante a integração dos indicadores de qualidade dos solos em Índices de Qualidade do Solo (IQS), obtidos por meio de uma expressão ou modelo matemático que inclua todos os atributos do solo considerados. Os valores ideais para os indicadores de qualidade de solos de florestas plantadas e manejadas seriam aqueles encontrados em florestas não-manejadas (naturais), consideradas auto-sustentáveis numa maior escala de tempo (Tótola \& Chaer, 2002).

Este trabalho objetivou acompanhar possíveis mudanças na qualidade de solos em povoamentos de eucalipto, mediante a utilização de irrigação e fertirrigação de efluente de uma fábrica de celulose, por meio de um modelo matemático que expressa o índice de qualidade do solo (IQS) considerando atributos microbiológicos e químicos.

\section{MATERIAL E MÉTODOS}

O estudo foi realizado em dois experimentos implantados em outubro de 2001, na estação experimental da Celulose Nipo-Brasileira S.A. (CENIBRA), na unidade de Belo Oriente, MG, localizada a $19^{\circ} 17^{\prime} 52^{\prime \prime S}$ e $42^{\circ} 23^{\prime} 24 " W$, na altitude de $214 \mathrm{~m}$. O clima da região é do tipo Aw, tropical chuvoso, com inverno seco e verão chuvoso, com precipitação média anual de $1163 \mathrm{~mm}$ e temperatura média anual de $25,2{ }^{\circ} \mathrm{C}$, com o menor valor ocorrendo no mês de julho $\left(22^{\circ} \mathrm{C}\right)$ e o maior em janeiro-fevereiro $\left(27,6^{\circ} \mathrm{C}\right)$. Os experimentos foram instalados em florestas de eucalipto localizadas em áreas com relevo suavemente ondulado situadas em posição de baixada e encosta com solos classificados como Neossolo Flúvico e Latossolo Amarelo, respectivamente. Foram considerados os seguintes tratamentos: 1) irrigação até a máxima evapotranspiração (Irrigação); 2) fertirrigação até a máxima evapotranspiração com $100 \%$ da adubação recomendada, em função da curva de demanda nutricional para uma produtividade estimada de 36 $\mathrm{m}^{3}$ de madeira aos seis anos de idade (Irrigação Adubação); 3) irrigação com efluente de celulose (Irrigação - Efluente). Além destes considerou-se também os tratamentos controle com ausência de irrigação e de adubação (C1) e de efluente (C2).

Os tratamentos Irrigação e Irrigação Adubação e seus controles, foram instalados nas posições de encosta e baixada, em parcelas cultivadas com o clone 57 (um híbrido de procedência Rio Claro), plantados no espaçamento $3 \times 3,33 \mathrm{~m}$. O tratamento Irrigação - Efluente e seu controle foram instalados apenas na posição de baixada com 26 clones (teste clonal) plantados no espaçamento $3 \times 2 \mathrm{~m}$. Além das áreas de plantio de eucalipto considerou-se em ambos os experimentos duas outras consideradas como referência sendo uma de mata secundária e outra de pastagem. A mata secundária era composta com algumas espécies da mata atlântica, além de algumas árvores de eucalipto e a pastagem encontrava-se em estado elevado de degradação. $\mathrm{Na}$ encosta a cultura de eucalipto encontrava-se no quarto ciclo do cultivo, significando aproximadamente 30 anos de uso do solo, enquanto que na baixada foi implantada a irrigação do eucalipto em substituição a um plantio de eucalipto de aproximadamente dois anos em áreas anteriormente ocupadas por pastagem.

As coletas de solo foram realizadas em março/abril (período chuvoso) e agosto/setembro (período seco) do ano de 2003. Foram retiradas dez amostras simples de solo da camada de $0-10 \mathrm{~cm}$ para formar uma amostra composta, sendo cinco delas na região das linhas de irrigação (bulbo molhado) e cinco nas entrelinhas. Para as áreas sem irrigação (controles), mata e pastagem as dez amostras simples foram distribuídas aleatoriamente. Para cada tratamento localizado nas posições topográficas distintas durante as épocas estudadas foram estabelecidas três parcelas de amostragem, cada uma representando uma repetição. As amostras foram acondicionadas em sacos plásticos previamente identificados e mantidas sob refrigeração a $4{ }^{\circ} \mathrm{C}$ até o momento das análises. Os solos foram analisados para a determinação do $\mathrm{C}$ orgânico pelo método de Walkley e Black, além dos parâmetros: $\mathrm{pH}$ (em água); teores de $\mathrm{P}, \mathrm{K}$ (extraíveis, por Mehlich-1), $\mathrm{Ca}, \mathrm{Mg}$ e Al (trocáveis, por $\mathrm{KCl} 1 \mathrm{~mol} \mathrm{dm}^{-3}$ ) (Defelipo \& Ribeiro, 1981) (Tabelas 1 e 2). A capacidade de retenção de água foi determinada mediante o equivalente de umidade, ou seja, pela água retida a $1000 \mathrm{~g}$ de solo (EMBRAPA, 1997).

O carbono da biomassa microbiana (CBM) foi avaliado pelo método da Respiração Induzida pelo Substrato (RIS) (Anderson \& Domsch, 1978). O quociente microbiano (qMIC), foi estimado pela relação entre o CBM e o carbono orgânico do solo. A respiração basal foi avaliada pela medição do $\mathrm{CO}_{2}$ liberado por amostras de $10 \mathrm{~g}$ de solo com teor de umidade de $60 \%$ da capacidade de retenção de água, durante $8 \mathrm{~h}$, mantendo-se as amostras a 
SANTOS, C.A.P. et al. Atributos indicadores da água do solo...

$22{ }^{\circ} \mathrm{C}$, por meio de um respirômetro dotado de um leitor de infravermelho (Sable Systems, NE, USA). A partir dos valores da respiração e do CBM, determinou-se o quociente metabólico $\left(\mathrm{qCO}_{2}\right)$, que representa a taxa de respiração microbiana por unidade de biomassa (Anderson \& Domsch, 1985).

TABELA 1 - Atributos químicos de Neossolo Flúvico com plantio de eucalipto submetido a diferentes manejos, localizado em baixada.

\begin{tabular}{|c|c|c|c|c|c|c|c|c|}
\hline Atributos & Período & \multicolumn{7}{|c|}{ Manejo $^{(1)}$} \\
\hline \multirow[b]{3}{*}{$\mathrm{pH} \mathrm{H} \mathrm{H}_{2} \mathrm{O}$} & & C1 & I & $\mathrm{IA}$ & $\mathrm{C} 2$ & IE & $\mathrm{P}$ & $M$ \\
\hline & Chuvoso & $5,42 \mathrm{Ca}^{(2)}$ & $5,55 \mathrm{Ca}$ & $5,66 \mathrm{BCa}$ & $6,16 \mathrm{ABa}$ & $6,27 \mathrm{Aa}$ & $5,52 \mathrm{Ca}$ & $5,15 \mathrm{Ca}$ \\
\hline & Seco & 5,53 Aba & $\begin{array}{l}5,12 \\
\text { BCb }\end{array}$ & $5,73 \mathrm{Aa}$ & 5,99 Aa & $5,71 \mathrm{Ab}$ & $5,42 \mathrm{ABa}$ & $4,80 \mathrm{Cb}$ \\
\hline \multirow{2}{*}{$\begin{array}{c}\mathrm{MO} \\
\left(\mathrm{dag} \mathrm{dm}^{-3}\right)\end{array}$} & Chuvoso & $1,72 \mathrm{Ba}$ & $1,73 \mathrm{Ba}$ & $1,64 \mathrm{Ba}$ & $2,13 \mathrm{Ba}$ & $2,01 \mathrm{Ba}$ & 3,35 Aa & $3,32 \mathrm{Aa}$ \\
\hline & Seco & 1,76 Ba & $1,66 \mathrm{Ba}$ & 1,85 Ba & $2,14 \mathrm{Ba}$ & 1,78 Ba & 3,37 Aa & $3,51 \mathrm{Aa}$ \\
\hline \multirow{2}{*}{$\begin{array}{c}\mathrm{P} \\
\left.(\mathrm{mg} \mathrm{dm})^{-3}\right)\end{array}$} & Chuvoso & $86,6 \mathrm{Ab}$ & $17,10 \mathrm{Aa}$ & $36,1 \mathrm{Aa}$ & $115,4 \mathrm{Aa}$ & $46,6 \mathrm{Aa}$ & $6,50 \mathrm{Aa}$ & $4,1 \mathrm{Aa}$ \\
\hline & Seco & $222,25 \mathrm{Aa}$ & $7,08 \mathrm{Ba}$ & $14,29 \mathrm{Ba}$ & 7,92 Ba & $8,95 \mathrm{Ba}$ & $2,54 \mathrm{Ba}$ & $5,40 \mathrm{Ba}$ \\
\hline \multirow{2}{*}{$\begin{array}{c}\mathrm{K} \\
\left.(\mathrm{mg} \mathrm{dm})^{-3}\right)\end{array}$} & Chuvoso & $79,5 \mathrm{Ba}$ & $72,7 \mathrm{Ba}$ & $117,4 \mathrm{Aba}$ & $135,6 \mathrm{ABa}$ & $135,6 \mathrm{ABa}$ & $183,6 \mathrm{Aa}$ & $113,7 \mathrm{ABa}$ \\
\hline & Seco & $58,17 \mathrm{Ba}$ & $51,50 \mathrm{Ba}$ & $104,83 \mathrm{Ba}$ & $127,84 \mathrm{ABa}$ & $103,17 \mathrm{Ba}$ & $208,50 \mathrm{Aa}$ & $102,50 \mathrm{Ba}$ \\
\hline \multirow{2}{*}{$\begin{array}{c}\mathrm{Ca} \\
\left(\mathrm{cmol}_{\mathrm{c}} \mathrm{dm}^{-3}\right)\end{array}$} & Chuvoso & 2,15 BCa & $1,95 \mathrm{Ca}$ & $2,32 \mathrm{ABCa}$ & $3,69 \mathrm{ABa}$ & 3,98 Aa & $2,25 \mathrm{BCa}$ & $2,35 \mathrm{ABCa}$ \\
\hline & Seco & $2,04 \mathrm{Aa}$ & $1,47 \mathrm{Aa}$ & $2,43 \mathrm{Aa}$ & $2,89 \mathrm{Aa}$ & $1,92 \mathrm{Ab}$ & $2,24 \mathrm{Aa}$ & $1,73 \mathrm{Aa}$ \\
\hline \multirow{2}{*}{$\begin{array}{c}\mathrm{Mg} \\
\left(\mathrm{cmol}_{\mathrm{c}} \mathrm{dm}^{-3}\right)\end{array}$} & Chuvoso & $0,88 \mathrm{ABa}$ & $0,79 \mathrm{ABa}$ & 0,86 Aba & $1,27 \mathrm{Aa}$ & $1,13 \mathrm{ABa}$ & 0,71 Ba & $0,66 \mathrm{Ba}$ \\
\hline & Seco & $0,77 \mathrm{Aa}$ & $0,81 \mathrm{Aa}$ & 1,17 Aa & $1,17 \mathrm{Aa}$ & $0,85 \mathrm{Aa}$ & $0,98 \mathrm{Aa}$ & $0,70 \mathrm{Aa}$ \\
\hline \multirow{2}{*}{$\frac{\mathrm{Al}}{\left(\mathrm{cmol}_{\mathrm{c}} \mathrm{dm}^{-3}\right)}$} & Chuvoso & $0,29 \mathrm{ABa}$ & $0,18 \mathrm{ABa}$ & $0,03 \mathrm{Ba}$ & $0,01 \mathrm{Ba}$ & $0,04 \mathrm{Ba}$ & $0,26 \mathrm{ABa}$ & $0,55 \mathrm{Aa}$ \\
\hline & Seco & $0,08 \mathrm{Ba}$ & $0,19 \mathrm{Ba}$ & $0,01 \mathrm{Ba}$ & $0,00 \mathrm{Ba}$ & $0,00 \mathrm{Ba}$ & $0,13 \mathrm{Ba}$ & $0,76 \mathrm{Aa}$ \\
\hline
\end{tabular}

(1) C1 - controle da irrigação e irrigação - adubação; I - irrigação; IA - irrigação - adubação; C2 - controle do efluente; IE - irrigação efluente; $\mathrm{P}$ - pastagem; $\mathrm{M}$ - mata secundária.

(2) Letras maiúsculas na linha comparam o efeito dos tratamentos numa mesma época, pelo teste de Tukey a $5 \%$ de probabilidade. Letras minúsculas na coluna comparam o efeito da época sobre cada tratamento, pelo teste $\mathrm{F}$ a $5 \%$ de probabilidade.

TABELA 2 - Atributos químicos de Latossolo Amarelo com plantio de eucalipto submetido a diferentes manejos, localizados em encosta.

\begin{tabular}{|c|c|c|c|c|c|c|}
\hline \multirow[t]{2}{*}{ Atributos } & \multirow[t]{2}{*}{ Período } & \multicolumn{5}{|c|}{ Manejo $^{(1)}$} \\
\hline & & $\mathrm{C} 1$ & 1 & $\mathrm{IA}$ & $\mathrm{P}$ & $\mathrm{M}$ \\
\hline \multirow{2}{*}{$\mathrm{pH} \mathrm{H} \mathrm{H}_{2} \mathrm{O}$} & Chuvoso & $6,32 \mathrm{Aa}^{(2)}$ & $4,74 \mathrm{BCa}$ & $4,82 \mathrm{Ba}$ & $4,74 \mathrm{Ba}$ & $4,34 \mathrm{Ca}$ \\
\hline & Seco & $5,95 \mathrm{Ab}$ & $4,90 \mathrm{Ba}$ & $4,79 \mathrm{Ba}$ & 4,67 Ba & $4,09 \mathrm{Ca}$ \\
\hline \multirow{2}{*}{$\begin{array}{c}\mathrm{MO} \\
\left(\mathrm{dag} \mathrm{dm}^{-3}\right)\end{array}$} & Chuvoso & $2,98 \mathrm{Aa}$ & 2,16 BCa & $1,75 \mathrm{Ca}$ & $2,76 \mathrm{ABa}$ & $2,84 \mathrm{Aa}$ \\
\hline & Seco & $2,71 \mathrm{Ab}$ & $2,37 \mathrm{Aa}$ & 1,66 Ba & $2,86 \mathrm{Aa}$ & $2,96 \mathrm{Aa}$ \\
\hline \multirow{2}{*}{$\begin{array}{c}P \\
\left(m g ~ d m^{-3}\right)\end{array}$} & Chuvoso & $4,46 \mathrm{Aa}$ & $4,12 \mathrm{Aa}$ & 3,99 Aa & $5,81 \mathrm{Aa}$ & $25,73 \mathrm{Aa}$ \\
\hline & Seco & $1,48 \mathrm{Aa}$ & $13,71 \mathrm{Aa}$ & $3,28 \mathrm{Aa}$ & $1,80 \mathrm{Aa}$ & $2,85 \mathrm{Aa}$ \\
\hline \multirow{2}{*}{$\begin{array}{c}\mathrm{K} \\
\left.(\mathrm{mg} \mathrm{dm})^{-3}\right)\end{array}$} & Chuvoso & $116,20 \mathrm{Aa}$ & $31,56 \mathrm{Ba}$ & $53,13 \mathrm{Ba}$ & $49,47 \mathrm{Ba}$ & $50,47 \mathrm{Ba}$ \\
\hline & Seco & $79,83 \mathrm{Ab}$ & $24,5 \mathrm{Ca}$ & $49,17 \mathrm{Ba}$ & $48,17 \mathrm{Ba}$ & $40,17 \mathrm{BCa}$ \\
\hline \multirow{2}{*}{$\begin{array}{c}\mathrm{Ca} \\
\left(\mathrm{cmol}_{\mathrm{c}} \mathrm{dm}^{-3}\right)\end{array}$} & Chuvoso & $5,75 \mathrm{Aa}$ & $1,11 \mathrm{Ba}$ & $1,12 \mathrm{Ba}$ & $1,24 \mathrm{Ba}$ & $0,10 \mathrm{Ca}$ \\
\hline & Seco & $5,22 \mathrm{Ab}$ & 1,18 Ba & $1,10 \mathrm{Ba}$ & $0,76 \mathrm{BCa}$ & $0,12 \mathrm{Ca}$ \\
\hline \multirow{2}{*}{$\begin{array}{c}\mathrm{Mg} \\
\left(\mathrm{cmol}_{\mathrm{c}} \mathrm{dm}^{-3}\right)\end{array}$} & Chuvoso & $0,97 \mathrm{Ab}$ & $0,32 \mathrm{BCb}$ & $0,26 \mathrm{BCa}$ & $0,35 \mathrm{Ba}$ & 0,01 Ca \\
\hline & Seco & $1,22 \mathrm{Aa}$ & $0,58 \mathrm{Ba}$ & $0,39 \mathrm{Ba}$ & $0,37 \mathrm{BCa}$ & $0,07 \mathrm{Ca}$ \\
\hline \multirow{2}{*}{$\begin{array}{c}\mathrm{Al} \\
\left(\mathrm{cmol}_{\mathrm{c}} \mathrm{dm}^{-3}\right)\end{array}$} & Chuvoso & 0,01 Ca & 0,68 Ba & $0,53 \mathrm{Ba}$ & $0,51 \mathrm{Ba}$ & 1,22 Aa \\
\hline & Seco & $0,002 \mathrm{Ba}$ & $0,44 \mathrm{Ba}$ & $0,273 \mathrm{Ba}$ & $0,437 \mathrm{Ba}$ & $1,263 \mathrm{Aa}$ \\
\hline
\end{tabular}

(1) C1 - controle da irrigação e irrigação - adubação; I - irrigação; IA - irrigação - adubação; P - pastagem; M - mata secundária.

(2) Letras maiúsculas na linha comparam o efeito dos tratamentos numa mesma época, pelo teste de Tukey a $5 \%$ de probabilidade. Letras minúsculas na coluna comparam o efeito da época sobre cada tratamento, pelo teste $\mathrm{F}$ a $5 \%$ de probabilidade. 
Foram avaliadas as atividades das seguintes enzimas do solo: fosfatase ácida e âglicosidase, utilizando-se os métodos descritos por Tabatabai (1994) e Eivazi \& Tabatabai (1988), respectivamente.

A taxa de amonificação líquida foi determinada segundo o método descrito por Bundy \& Meisinger (1994), para obtenção de um extrato filtrado em papel-filtro comum e guardado em geladeira para posterior análise dos teores de $\mathrm{NH}_{4}{ }^{+}$, nos tempos $\mathrm{t}=0$ e $\mathrm{t}=7$ dias. Determinou-se a taxa de nitrificação potencial empregando o método proposto por Belser \& Mays (1980), descrito em Hart et al. (1994). A taxa de produção de $\mathrm{N}^{-\mathrm{NO}_{3}}{ }_{3}^{-}$foi calculada segundo metodologia descrita por Yang et al. (1998).

Os dados obtidos por meio da integração dos indicadores microbiológicos e químicos foram utilizados para cálculo do IQS, utilizando-se o programa SIMOQS (Sistema de Monitoramento da Qualidade do Solo), desenvolvido por Chaer (2001) e submetidos às análises de variância em cada posição topográfica separadamente, realizadas com o uso do software SAEG 5.0 (FUNARBE, 1993). Adotou-se o delineamento inteiramente casualizado em esquema de parcelas subdivididas com três repetições considerando sete sistemas de manejo nas parcelas para a posição de baixada (Neossolo Flúvico) e cinco para a posição de encosta (Latossolo Amarelo), ambos com duas épocas de amostragem nas subparcelas (período seco e chuvoso). A comparação das médias foi feita no sentido de avaliar o efeito do tratamento dentro de cada época pelo teste de Tukey a $5 \%$ e da época dentro de cada tratamento pelo teste $\mathrm{F}$ a $5 \%$.

\section{RESULTADOS E DISCUSSÃO}

Os maiores teores de matéria orgânica foram encontrados nos tratamentos de mata e pastagem em ambas as posições topográficas e períodos climáticos (Tabelas 1 e 2). Por outro lado, a implantação de eucalipto na área de baixada resultou em redução acentuada da matéria orgânica (Tabela 1). Considerando que no Brasil, as florestas de eucaliptos começam a depositar serapilheira somente a partir do terceiro ano (Monteiro \& GamaRodrigues, 2004), entende-se que, nesses povoamentos de eucalipto (com apenas dois anos de idade), o teor de matéria orgânica do solo se eleve com o aumento da idade da floresta.

A atividade biológica nos solos é limitada pela quantidade de carbono prontamente disponível. A redução da biomassa microbiana no tratamento Irrigação - Adubação e em ambas as posições topográficas no período chuvoso ou no tratamento Irrigação - Efluente foi atribuída ao baixo teor de carbono orgânico nos solos desses tratamentos (Tabelas 3 e 4). O baixo valor de CBM no tratamento Irrigação - Adubação na encosta relaciona-se com a redução do teor de matéria orgânica, aparentemente como o resultado do aporte contínuo de nutrientes minerais. Assim, ao contrário do observado por Chaer (2001) e Lazari (2001) que demonstraram a diminuição do CBM em função da implantação de povoamentos de eucalipto, o CBM das áreas estudadas neste trabalho não sofreu nenhum tipo de redução (Tabelas 3 e 4), comparativamente aos valores encontrados na área de referência (solos sob mata natural). Esta situação foi mais evidente durante o período seco, comportamento que se reflete também na proporção de CBM na matéria orgânica do solo (qMIC).

$\mathrm{Na}$ posição de baixada, os tratamentos de eucalipto apresentaram baixos valores de $\mathrm{qCO}_{2}$, o que indica a eficiência da comunidade microbiana nestes solos quanto à utilização de substratos orgânicos pois, quando uma maior fração de carbono está sendo incorporada à biomassa, menos carbono está sendo perdido pela respiração, em ambos os períodos estudados. A alta atividade de respiração basal encontrada nos tratamentos de mata e pastagem na posição de baixada, nos dois períodos estudados, pode representar um indicativo de estresse, reforçado pelos altos valores de $\mathrm{qCO}_{2}$ obtidos nessas áreas. Esses dados são condizentes com os baixos valores de quociente microbiano (qMIC) nesses solos, evidenciando a menor participação da biomassa microbiana na matéria orgânica do solo (Tabela 3 ).

A alta atividade da fosfatase ácida em solos cultivados com eucalipto, nas duas posições topográficas e em ambos os períodos climáticos (Tabelas 3 e 4), evidenciou a importância das formas orgânicas de $P$ no fornecimento deste elemento às plantas, também observados por Conte et al. (2002), que observaram maiores teores de fósforo orgânico e de atividade da fosfatase ácida em solos com vegetação nativa. $\mathrm{Na}$ baixada durante o período de maior restrição hídrica, houve redução da atividade de â-glicosidase em solos sob eucalipto. $\mathrm{Na}$ encosta, esse comportamento não foi observado (Tabelas 3 e 4). Analisando-se os dados do $\mathrm{qCO}_{2}$ com a atividade da enzima â-glicosidase, durante o período seco na posição de baixada, observa-se que ecossistemas não-manejados (mata e pastagem) apresentam estreita relação entre a atividade enzimática do solo e a produção de biomassa vegetal. Embora não tenha sido feita comparação entre períodos quanto às atividades de â-glicosidase e fosfatase ácida em decorrência da grande heterogeneidade dos valores obtidos da variância do erro, os menores valores encontrados no período chuvoso podem ser resultado de maior tempo de armazenamento das amostras coletadas nessa época.

As taxas de amonificação líquida foram maiores nos tratamentos sob mata e pastagem na posição de baixada, o que pode ser atribuído ao maior teor de matéria orgânica nesses solos nos dois períodos climáticos estudados (Tabelas 3 e 4). Esses dados são semelhantes aos resultados descritos por Chaer (2001) e Lazari (2001). A atividade de nitrificação potencial durante o período chuvoso foi significativamente superior à obtida no período seco, época em que os tratamentos (Irrigação e Irrigação - Efluente e seus respectivos 
SANTOS, C.A.P. et al. Atributos indicadores da água do solo...

TABELA 3 - Atributos microbiológicos de Neossolo Flúvico com plantio de eucalipto submetido a diferentes manejos, localizado em baixada.

\begin{tabular}{|c|c|c|c|c|c|c|c|c|}
\hline Atributos & Período & & & & Manejo & & & \\
\hline & & C1 & I & IA & $\mathrm{C} 2$ & IE & $\mathrm{P}$ & $\mathrm{M}$ \\
\hline $\begin{array}{l}\text { Carbono da } \\
\text { Biomassa }\end{array}$ & Chuvoso & $270,0 \mathrm{Aa}^{(2)}$ & $221,0 \mathrm{Aba}$ & $113,0 \mathrm{Bb}$ & $124,0 \mathrm{Ba}$ & $110,0 \mathrm{Bb}$ & $163,0 \mathrm{ABb}$ & $154,0 \mathrm{Aba}$ \\
\hline $\begin{array}{c}\text { Microbiana } \\
\text { (ug CBM g }{ }^{-1} \text { solo) }\end{array}$ & Seco & 203,0 Aa & 193,0 Aa & $207,0 \mathrm{Aa}$ & $177,0 \mathrm{Aa}$ & 196,0 Aa & $275,0 \mathrm{Aa}$ & $187,0 \mathrm{Aa}$ \\
\hline Quociente & Chuvoso & $2,742 \mathrm{Aa}$ & $2,214 \mathrm{Aba}$ & 1,189 BCb & 1,033 Ca & $0,951 \mathrm{Cb}$ & $0,859 \mathrm{Ca}$ & 0,801 Ca \\
\hline$(\%)$ & Seco & $2,016 \mathrm{Ab}$ & $2,036 \mathrm{Aa}$ & 1,948 ABa & 1,407 Aba & $1,952 \mathrm{ABa}$ & 1,466 ABa & 0,915 Ba \\
\hline $\begin{array}{c}\text { Respiração } \\
\text { basal }\end{array}$ & Chuvoso & $0,71 \mathrm{Ba}$ & $0,65 \mathrm{Bb}$ & $0,64 \mathrm{Bb}$ & $0,49 \mathrm{Bb}$ & 0,89 Ba & $3,27 \mathrm{Ab}$ & 2,61 Aa \\
\hline$\left(u g \mathrm{CO}_{2} \mathrm{~g}^{-1} \mathrm{~h}^{-1}\right)$ & Seco & $1,11 \mathrm{Ca}$ & $1,48 \mathrm{Ca}$ & $1,54 \mathrm{Ca}$ & $1,60 \mathrm{Ca}$ & $1,17 \mathrm{Ca}$ & $4,47 \mathrm{Aa}$ & $3,29 \mathrm{Ba}$ \\
\hline $\begin{array}{l}\text { Quociente } \\
\text { metabólico }\end{array}$ & Chuvoso & $0,0026 \mathrm{Ca}$ & $0,0031 \mathrm{Cb}$ & 0,0058 Ca & $0,0039 \mathrm{Cb}$ & $0,0086 \mathrm{BCa}$ & 0,0203 Aa & 0,0168 Aba \\
\hline$\left(u g \mathrm{CO}_{2} \mathrm{~h}^{-1} u \mathrm{~g}^{-1} \mathrm{CBM}\right)$ & Seco & $0,0055 \mathrm{Ca}$ & $0,0077 \mathrm{Ca}$ & $0,0075 \mathrm{Ca}$ & $0,009 \mathrm{BCa}$ & $0,0060 \mathrm{Ca}$ & 0,0177 ABa & $0,0182 \mathrm{Aa}$ \\
\hline $\begin{array}{c}\text { Fosfatase } \\
\text { Ácida* }\end{array}$ & Chuvoso & $1733,0 \mathrm{~A}$ & $1143,0 \mathrm{~A}$ & $1022,0 \mathrm{~A}$ & $892,0 \mathrm{~A}$ & $887,0 \mathrm{~A}$ & $1248,0 \mathrm{~A}$ & $1878,0 \mathrm{~A}$ \\
\hline (ug $g^{-1} \mathrm{~h}^{-1} p$-nitrofenol) & Seco & $5886,0 \mathrm{~A}$ & $6941,0 \mathrm{~A}$ & $11113,0 \mathrm{~A}$ & $6076,0 \mathrm{~A}$ & $14994 \mathrm{~A}$ & $8584,0 \mathrm{~A}$ & $5070,0 \mathrm{~A}$ \\
\hline$\beta$-glicosidase* & Chuvoso & $162,0 \mathrm{BC}$ & $174,0 \mathrm{BC}$ & $200,0 \mathrm{ABC}$ & $190,0 \mathrm{ABC}$ & $147,0 \mathrm{C}$ & $281,0 \mathrm{~A}$ & $247,0 \mathrm{AB}$ \\
\hline (ug $\mathrm{g}^{-1} \mathrm{~h}^{-1} p$-nitrofenol) & Seco & $544,0 \mathrm{~B}$ & $514,0 \mathrm{~B}$ & $420,0 \mathrm{~B}$ & $598,0 \mathrm{~B}$ & $471,0 \mathrm{~B}$ & $1340,0 \mathrm{~A}$ & $1245,0 \mathrm{~A}$ \\
\hline $\begin{array}{c}\text { Amonificação } \\
\text { Líquida }\end{array}$ & Chuvoso & $1,48 \mathrm{Ba}$ & $1,83 \mathrm{Ba}$ & $1,68 \mathrm{Ba}$ & $1,42 \mathrm{Ba}$ & 1,57 Ba & 4,34 Aa & $4,14 \mathrm{Ab}$ \\
\hline$\left(u g \mathrm{~N}-\mathrm{NH}_{4} \mathrm{~g}^{-1} \mathrm{dia}^{-1}\right)$ & Seco & $2,38 \mathrm{Ca}$ & $2,46 \mathrm{Ca}$ & 2,81 Ca & $2,34 \mathrm{Ca}$ & $2,78 \mathrm{Ca}$ & $5,27 \mathrm{Ba}$ & $7,40 \mathrm{Aa}$ \\
\hline $\begin{array}{c}\text { Nitrificação } \\
\text { Potencial }\end{array}$ & Chuvoso & 7,4 Aa & 7,1 Aa & $11,1 \mathrm{Aa}$ & $13,3 \mathrm{Aa}$ & $15,9 \mathrm{Aa}$ & 2,6 Aa & 7,2 Aa \\
\hline$\left(u g \mathrm{~N}-\mathrm{NO}_{3}^{-} \mathrm{g}^{-1} \mathrm{ss} \mathrm{h}^{-1}\right)$ & Seco & $-6,3 \mathrm{Ab}$ & $-3,8 \mathrm{Ab}$ & $-2,0 \mathrm{Aa}$ & $1,9 \mathrm{Ab}$ & $-0,6 \mathrm{Ab}$ & $-2,5 \mathrm{Aa}$ & $2,1 \mathrm{Aa}$ \\
\hline \multirow{2}{*}{$\mathrm{IQS}^{(3)}$} & Chuvoso & $95,89 \mathrm{ABa}$ & $97,54 \mathrm{Aa}$ & $92,98 \mathrm{ABCb}$ & $94,45 \mathrm{ABa}$ & $90,92 \mathrm{ABCb}$ & $87,20 \mathrm{Ca}$ & $89,20 \mathrm{BCa}$ \\
\hline & Seco & $97,35 \mathrm{Aa}$ & $96,48 \mathrm{Aa}$ & $97,59 \mathrm{Aa}$ & $95,83 \mathrm{ABa}$ & $97,69 \mathrm{Aa}$ & $90,74 \mathrm{ABa}$ & $89,10 \mathrm{Ba}$ \\
\hline
\end{tabular}

${ }^{(1)}$ C1 - controle da irrigação e irrigação - adubação; I - irrigação; IA - irrigação - adubação; C2 - controle do efluente; IE - irrigação efluente; $\mathrm{P}$ - pastagem; $\mathrm{M}$ - mata secundária.

(2) Letras maiúsculas na linha comparam o efeito dos tratamentos numa mesma época, pelo teste de Tukey a $5 \%$ de probabilidade. Letras minúsculas na coluna comparam o efeito da época sobre cada tratamento, pelo teste $\mathrm{F}$ a $5 \%$ de probabilidade.

(3) O IQS estabelecido considera ainda os atributos químicos, porém para efeito de demonstração, a sua inclusão foi feita nos atributos microbiológicos.

controles) apresentaram nitrificação potencial negativa (Tabela 3). Normalmente a taxa de nitrificação potencial apresenta variação temporal, ou melhor, uma estreita relação entre a concentração de $\mathrm{NO}_{3}^{-}$e a pluviosidade. Assim, durante o período seco, há baixa taxa de mineralização, porém, com o início das chuvas, o aumento da taxa de mineralização da matéria orgânica propicia maior liberação de $\mathrm{NH}_{4}^{+}$, substrato da nitrificação e, conseqüentemente, aumento da concentração de $\mathrm{NO}_{3}^{-}$no solo (Wheatley et al., 2001).

A integração dos valores dos indicadores analisados em um modelo de determinação de um índice de qualidade de solos (Chaer, 2001) permitiu distinguir a qualidade dos solos amostrados em diferentes posições topográficas e épocas climáticas. Os tratamentos referentes à Irrigação-Adubação e Irrigação - Efluente, na posição de baixada, apresentaram menor IQS durante o período chuvoso
(Tabela 3), fato atribuído ao excesso de água no solo, além da baixa eficiência de uso da água na produção de biomassa. Na posição de encosta e no período seco, os tratamentos controle da irrigação, Irrigação - Adubação e mata apresentaram os menores IQS quando comparado o efeito de época (Tabela 4). Esse comportamento pode ser atribuído ao fato de que a posição de encosta pode estar mais suscetível a uma situação de estresse ocasionado pela escassez de água ou nutrientes necessários à atividade das comunidades microbianas. Já o comportamento diferenciado do IQS na posição de baixada possivelmente deve-se ao fato que nos solos dessas áreas houve o efeito de época nos diferentes tratamentos, que durante o período chuvoso ocorre encharcamento do solo, dada a baixa profundidade do lençol freático. 
SANTOS, C.A.P. et al. Atributos indicadores da água do solo...

TABELA 4 - Atributos microbiológicos de Latossolo Amarelo com plantio de eucalipto submetido a diferentes manejos, localizado em encosta.

\begin{tabular}{|c|c|c|c|c|c|c|}
\hline Atributos & Período & & & Manejo $^{(1)}$ & & \\
\hline \multirow{3}{*}{$\begin{array}{l}\text { Carbono da biomassa microbiana } \\
\qquad\left(\text { ug CBM g }{ }^{-1} \text { solo }\right)\end{array}$} & & $\mathrm{C} 1$ & I & IA & $\mathrm{P}$ & $M$ \\
\hline & Chuvoso & $187,0 \mathrm{ABa}^{(2)}$ & $185,0 \mathrm{ABa}$ & $139,0 \mathrm{Ba}$ & $262,0 \mathrm{Aa}$ & $209,0 \mathrm{Aba}$ \\
\hline & Seco & $87,0 \mathrm{Ab}$ & $119,0 \mathrm{Aa}$ & $80,0 \mathrm{Aa}$ & $164,0 \mathrm{Ab}$ & $137,0 \mathrm{Aa}$ \\
\hline \multirow{2}{*}{$\begin{array}{l}\text { Quociente microbiano } \\
\qquad(\%)\end{array}$} & Chuvoso & $1,861 \mathrm{Aa}$ & $1,059 \mathrm{Ba}$ & 1,121 Ba & $1,634 \mathrm{ABa}$ & 1,277 Aba \\
\hline & Seco & $0,902 \mathrm{Ab}$ & $0,760 \mathrm{Aa}$ & $0,586 \mathrm{Ab}$ & $1,002 \mathrm{Ab}$ & $0,816 \mathrm{Ab}$ \\
\hline \multirow{2}{*}{$\begin{array}{l}\text { Respiração basal } \\
\left(u g \mathrm{CO}_{2} \mathrm{~g}^{-1} \mathrm{~h}^{-1}\right)\end{array}$} & Chuvoso & $1,08 \mathrm{Ba}$ & $1,30 \mathrm{Ba}$ & $1,92 \mathrm{ABa}$ & 2,39 Aa & $1,70 \mathrm{Aba}$ \\
\hline & Seco & $1,09 \mathrm{Ca}$ & $1,33 \mathrm{BCa}$ & $2,00 \mathrm{ABCa}$ & $2,84 \mathrm{Aa}$ & 2,21 Aba \\
\hline \multirow{2}{*}{$\begin{array}{l}\text { Quociente metabólico } \\
\left.\text { (ug } \mathrm{CO}_{2} \mathrm{~h}^{-1} u^{-1} \mathrm{CBM}\right)\end{array}$} & Chuvoso & $0,0073 \mathrm{Ab}$ & $0,0121 \mathrm{Aa}$ & 0,0077 Aa & $0,0095 \mathrm{Ab}$ & $0,0081 \mathrm{Ab}$ \\
\hline & Seco & $0,0153 \mathrm{Aa}$ & 0,0168 Aa & $0,0136 \mathrm{Aa}$ & $0,0172 \mathrm{Aa}$ & $0,0161 \mathrm{Aa}$ \\
\hline \multirow{2}{*}{$\begin{array}{c}\text { Fosfatase ácida * } \\
\text { (ug g } \mathrm{g}^{-1} \mathrm{~h}^{-1} p \text {-nitrofenol) }\end{array}$} & Chuvoso & $668,0 \mathrm{~B}$ & $1022,0 \mathrm{AB}$ & $1427,0 \mathrm{~A}$ & $833,0 \mathrm{AB}$ & $1268,0 \mathrm{AB}$ \\
\hline & Seco & $4023,0 \mathrm{~B}$ & $10552,0 \mathrm{AB}$ & $6108,0 \mathrm{AB}$ & $8683,0 \mathrm{AB}$ & $13828,0 \mathrm{~A}$ \\
\hline \multirow{2}{*}{$\begin{array}{c}\beta \text {-glicosidase * } \\
\text { (ug g }{ }^{-1} h^{-1} p \text {-nitrofenol) }\end{array}$} & Chuvoso & $206,0 \mathrm{AB}$ & $166,0 \mathrm{~B}$ & $204,0 \mathrm{AB}$ & $247,0 \mathrm{~A}$ & $226,0 \mathrm{AB}$ \\
\hline & Seco & $824,0 \mathrm{~A}$ & $533,0 \mathrm{~A}$ & $544,0 \mathrm{~A}$ & $995,0 \mathrm{~A}$ & $845,0 \mathrm{~A}$ \\
\hline \multirow{2}{*}{$\begin{array}{l}\text { Amonificação Líquida } \\
\left(\text { ug N-NH} 4 \mathrm{~g}^{-1} \text { dia }^{-1}\right)\end{array}$} & Chuvoso & 1,87 Aba & $1,37 \mathrm{Ba}$ & $1,36 \mathrm{Bb}$ & $2,27 \mathrm{ABb}$ & $3,0 \mathrm{Aa}$ \\
\hline & Seco & $1,61 \mathrm{Ba}$ & $2,44 \mathrm{ABa}$ & $3,56 \mathrm{Aa}$ & 3,5 Aa & 2,67 Aba \\
\hline \multirow{2}{*}{$\begin{array}{l}\text { Nitrificação Potencial } \\
\left(u g \mathrm{~N}^{-N^{-}} \mathrm{g}^{-1} \mathrm{ss} \mathrm{h}^{-1}\right)\end{array}$} & Chuvoso & 5,6 Aba & $9,4 \mathrm{Aa}$ & 1,7 Aba & $-5,2 \mathrm{Ba}$ & 6,9 Aa \\
\hline & Seco & $1,8 \mathrm{Aa}$ & $-6,7 \mathrm{Ab}$ & $0,5 \mathrm{Aa}$ & $-5,6 \mathrm{Aa}$ & $1,2 \mathrm{Aa}$ \\
\hline \multirow{2}{*}{$\mathrm{IQS}^{(3)}$} & Chuvoso & $95,46 \mathrm{Aa}$ & $90,11 \mathrm{Aa}$ & $92,17 \mathrm{Aa}$ & $93,78 \mathrm{Aa}$ & $91,16 \mathrm{Aa}$ \\
\hline & Seco & $85,24 A b$ & $87,23 \mathrm{Aa}$ & $84,32 \mathrm{Ab}$ & $88,38 \mathrm{Aa}$ & $84,42 \mathrm{Ab}$ \\
\hline
\end{tabular}

(1) C1 - controle da irrigação e irrigação - adubação; I - irrigação; IA - irrigação - adubação; P - pastagem; M - mata secundária.

(2) Letras maiúsculas na linha comparam o efeito dos tratamentos numa mesma época, pelo teste de Tukey a $5 \%$ de probabilidade. Letras minúsculas na coluna comparam o efeito da época sobre cada tratamento, pelo teste $\mathrm{F}$ a $5 \%$ de probabilidade.

(3) O IQS estabelecido considera ainda os atributos químicos, porém para efeito de demonstração, a sua inclusão foi feita nos atributos microbiológicos.

\section{CONCLUSÕES}

1. Os atributos químicos mostraram-se mais sensíveis ao efeito do uso e manejo do solo que ao efeito de sazonalidade.

2. A biomassa microbiana foi influenciada pelo uso e manejo do solo, bem como pela posição topográfica e sazonalidade;

3. Os IQS apresentaram comportamento similar entre os solos de eucaliptos, mata e pastagem, apenas para a posição de encosta
(Latossolo Amarelo), mas não para a de baixada (Neossolo Flúvico).

\section{AGRADECIMENTOS}

Ao Conselho Nacional de Desenvolvimento Científico e Tecnológico ( $\mathrm{CNPq}$ ) pelo apoio através da concessão de bolsa e a Universidade Federal de Viçosa, especialmente ao Departamento de Microbiologia.

\section{REFERÊNCIAS}

1. ANDERSON, J. P. E.; DOMSCH, K. H. A physiological method for the quantitative measurement of microbial biomass in soils. Soil Biology and Biochemistry, v. 10, n. 3, p. 215-221, 1978.

2. ANDERSON, J. P. E.; DOMSCH, K. H. Determination of ecophysiological maintenance carbon requirements of soil microorganisms in a dormant state. Biology and Fertility of Soils, v. 1, n. 2, p. 81-89, 1985.

3. BELSER, L. W.; MAYS, E. L. Specific inhibition of nitrite oxidation by chlorate and its use in assessing nitrification in soils and sediments. Applied Environmental Microbiology, v. 39, n. 3, p. 505-510, 1980.

4. BUNDY, L. G.; MEISINGER, J. J. Nitrogen availability indices. In: WEAVER, R. W. et al. (Ed.). Methods of soil analysis: Part 2. Microbiological and biochemical properties, n. 5. Madison: Soil Science Society of America, 1994. p. 951-984. 
5. CHAER, G. M. Modelo para determinação de índice de qualidade do solo baseado em indicadores físicos, químicos e microbiológicos. 2001. 90 f. Dissertação (Mestrado em Microbiologia Agrícola) - Curso de Pós-Graduação em Microbiologia Agrícola, Setor de Ciências Biológicas, Universidade Federal de Viçosa, Viçosa, MG, 2001.

6. CONTE, E.; ANGHINONI, L.; RHEINHEIMER, D. S. Fósforo da biomassa microbiana e atividade de fosfatase ácida após aplicação de fosfato em solo no sistema plantio direto. Revista Brasileira de Ciência do Solo, v. 26, p. 925-930, 2002.

7. DEFELIPO, B. V.; RIBEIRO, A. C. Análise química do solo. Viçosa, MG: Universidade Federal de Viçosa, 1981. 17 p. (Boletim de extensão, 29).

8. EIVAZI, F.; TABATABAI, M. A. Glucosidases and galactosidases in soils. Soil Biology and Biochemistry, v. 20, n. 5, p. 601-606, 1988.

9. EMPRESA BRASILEIRA DE PESQUISA AGROPECUÁRIA (EMBRAPA). Manual de métodos de análise de solo. 2. ed. Rio de Janeiro: EMBRAPA-CNPS, 1997. $212 \mathrm{p}$.

10. FUNDAÇÃO ARTHUR BERNARDES (FUNARBE). SAEG - Sistema para análise estatística. V. 5.0. Viçosa, MG: UFV/ FUNARBE, 1993.

11. HART, S. C. et al. Nitrogen mineralization, immobilization and nitrification. In: WEAVER, R. W. et al. (Ed.). Methods of soil analysis: Part 2. Microbiological and biochemical properties. Madison: Soil Science Society of America, 1994. p. 9851018.

12. KELTING, D. L. et al. Soil quality assessment in domesticated forests-a southern pine example. Forest Ecology and Management, v. 122, n. 1/2, p. 167-185, 1999.

13. LAZARI, M. F. Nitrificação em solos sob plantações de eucalipto com diferentes idades. 2001. $50 \mathrm{f}$. Dissertação (Mestrado em Microbiologia Agrícola) - Curso de Pós-Graduação em Microbiologia Agrícola, Setor de Ciências Biológicas, Universidade Federal de Viçosa, Viçosa, MG, 2001.

14. LOPES, E. B. M. Diversidade metabólica em solo tratado com biossólidos. 2001. 65 f. Dissertação (Mestrado em Agronomia) - Curso de Pós-Graduação em Agronomia, Setor de Ciências Agrárias, Escola Superior de Agricultura "Luiz de Queiroz", Piracicaba, 2001.

15. MONTEIRO, M. T.; GAMA-RODRIGUES, E. F. Carbono, nitrogênio e atividade da biomassa microbiana em diferentes estruturas de serapilheira de uma floresta natural. Revista Brasileira de Ciência do Solo, v. 28, n. 5, p. 819-826, 2004.

16. OLIVEIRA, J. R. A.; MENDES, I. C.; VIVALDI, L. Carbono da biomassa microbiana em solos de cerrado sob vegetação nativa e sob cultivo: avaliação dos métodos fumigação-incubação e fumigação-extração. Revista Brasileira de Ciência do Solo, v. 25, n. 4, p. 863-871, 2001.

17. STENBERG, B. Monitoring soil quality of arable land: microbiological indicators. Soil and Plant Science, v. 49, p. 1-24, 1999.

18. TABATABAI, M. A. Soil enzymes. In: WEAVER, R. W. et al. (Ed.). Methods of soil analysis: Part 2. Microbiological and biochemical properties. Madison: Soil Science Society of America, 1994. p. 775-833.

19. TÓTOLA, M. R.; CHAER, G. M. Microrganismos e processos microbiológicos como indicadores de qualidade do solo. Tópicos Avançados em Ciência do Solo, v. 2, p. 195-276, 2002.

20. WHEATLEY, R. E. et al. Temporal variations in potential nitrification dynamics in soil related to differences in rates and types of carbon and nitrogen inputs. Soil Biology and Biochemistry, v. 33, n. 15, p. 2135-2144, 2001.

21. YANG, J. E. et al. A simple spectrophotometric determination of nitrate in water, resin, and soil extracts. Soil Science Society of America Journal, v. 62, p. 1108-1115, 1998. 
